\section{Revista de Investigación en Logopedia} ISSN: 1131-8635

http://dx.doi.org/10.5209/RLOG.62387

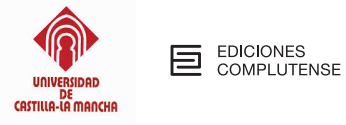

\title{
Efecto inmediato de la terapia de tracto vocal semiocluido en los parámetros acústicos en personas transexuales entre 13 a 24 años
}

\author{
María Soledad Sandoval Z. ${ }^{1}$; Rodrigo Fuenzalida C. ${ }^{2}$, Tanya Pérez Z. ${ }^{3}$; Francisco Torres Ch. ${ }^{4}$
}

Resumen. El presente estudio tiene como propósito describir el efecto inmediato de la terapia de tracto vocal semiocluido (TVSO) sobre los parámetros acústicos de personas transexuales entre 13 a 24 años. Es un estudio de tipo cuantitativo, con un diseño preexperimental con pre y post prueba, cuya dimensión de temporalidad es trasnversal y cuyo alcance es descriptivo. La muestra está constituida por 8 personas transexuales de 13 a 24 años de la comuna de Chillán, Chile, que no han recibido ningún tratamiento que pueda modificar sus características vocales. El instrumento utilizado para medir los parámetros acústicos es el programa de análisis acústico de la voz PRAAT (Boersma y Weenink, 2018). Los resultados indican que aunque los valores de los parámetros acústicos disminuyeron sólo existe un efecto inmediato en los parámetros acústicos Jitter Local y Jitter Rap, ya que muestra diferencias significativas antes y después de la terapia TVSO. De acuerdo a la perturbación de la frecuencia fundamendal, la voz de las participantes se hizo más grave y la relación armónico ruido se acercó al valor de normalidad luego de la terapia. Finalmente, se concluye que la terapia de TVSO tiene efecto inmediato en el parámetro acústico Jitter, en personas que transitan de sexo femenino a masculino de 13 a 24 años y puede considerarse un alternativa viable y complementaria en el proceso de masculinización de la voz. Palabras clave: parámetros acústicos; Población transexual; PRAAT; Terapia tracto vocal semiocluido.

\section{[en] Immediate effect of semioccluded vocal tract therapy on acoustic parameters in transsexuals between 13 and 24 years}

Abstract. The purpose of this study is to describe the immediate effects of semioccluded vocal tract therapy on the acoustic parameters of transsexuals between 13 and 24 years old. This is a quantitative study, with a preexperimental design with pre and post test, whose dimension of temporality is transverse and whose scope is descriptive. The sample is composed of 8 transsexuals from 13 to 24 years old from Chillán, who have not received any treatment that can modify their vocal characteristics. The instrument used to measure the acoustic parameters is the PRAAT program (Boersma y Weenink, 2018). The results indicate that although the values of the acoustic parameters decreased, there is only an immediate effect in the acoustic parameters Jitter Local and Jitter Rap, because they present statistically significant differences between the measurements made before and after the therapy. According to the perturbation of the fundamendal frequency, the transsexuals' voices became more deep and the harmo-

Facultad Ciencias de la Salud, Universidad Pedro de Valdivia, Sede Chillán.

Facultad Ciencias de la Salud, Universidad San Sebastián, Sede Concepción.

Núcleo de Investigación en Educación, Ciencias Sociales y Conservación del Medio Ambiente y Patrimonio de Ñuble, Universidad Adventista de Chile, Chillán.

solesandoval@hotmail.com

2 Facultad Ciencias de la Salud. Universidad Pedro de Valdivia, Chile.

3 Facultad Ciencias de la Salud. Universidad Pedro de Valdivia, Chile.

4 Facultad Ciencias de la Salud. Universidad Pedro de Valdivia, Chile. 
nics-to-noise ratio approached the normal value after the therapy. It is concluded that the semioccluided vocal tract therapy has immediate effect on the Jitter acoustic parameter in male-to-female transsexuals from 13 to 24 years old and this therapy can be considered a viable and complementary alternative in the process of masculinization of the voice.

Key Words: Acoustic parameters; PRAAT; Semioccluded vocal tract therapy; Transsexual.

Cómo citar: Sandoval Z., M.S.; Fuenzalida Z., R.; Pérz Z., T.; Torres Ch., F. (2019). Efecto inmediato de la terapia de tracto vocal semiocluido en los parámetros acústicos en personas transexuales entre 13 a 24 años. Revista de Investigación en Logopedia, 9(1), 1-19.

\section{Introducción}

La voz posee diferentes características y entre éstas se encuentran los parámetros acústicos, que han sido objeto de estudio en muchas investigaciones asociadas al área. El Jitter corresponde a la variación de la frecuencia fundamental entre un ciclo vocal y otro (Casado, 2002) y representa la estabilidad de la fonación (Cobeta, 2013); el valor normal del Jitter Local es de $0,63 \%$ y su valor límite $<1,040 \%$, mientras que el del Jitter Rap es de $0,63 \%$ con un valor límite $<0,680 \%$ y para el Jitter ppq 5 es $<0,840 \%$ (Cecconello, 2008). El Shimmer es la medida de perturbación de la amplitud que permite cuantificar pequeños lapsos de inestabilidad de la señal vocal (Jackson-Menaldi, 2002); los valores considerados de normalidad para el Shimmer local corresponden a $1,99 \%$ con un valor límite de $<3,810 \%$ y para el Shimmer apq11, el valor normal es de un 1,39\%, con un valor límite de $<3,070 \%$ (Cecconello, 2008). La frecuencia fundamental (F0) se define como el número de veces que las cuerdas vocales vibran por segundo (Casado, 2002); los valores de normalidad en hombres es de $119 \mathrm{~Hz}( \pm 20)$ y $207 \mathrm{~Hz}( \pm 24)$ en las mujeres (Elisei, 2012). Finalmente, la relación armónico-ruido (HNR, por su abreviación en inglés) mide el porcentaje de periodicidad o señal armónica y la aperiodicidad o ruido glótico en la onda vocal (Jackson-Menaldi, 1992; Lopes et al., 2008), siendo su rango de normalidad mayor a $20 \mathrm{~dB}$ (Cecconello, 2008).

En la actualidad, es sabido que una voz es considerada normal cuando presenta una altura tonal acorde a la edad y al sexo del hablante, una intensidad adecuada, un timbre agradable y una flexibilidad que derive del constate juego entre altura e intensidad (Farias, 2007). No obstante, existen muy pocos estudios que se centren en las características vocales de personas transexuales y la escasa información existente corresponden a investigaciones de personas que transitan del sexo masculino al femenino. Según Pérez (2017), la incidencia en estas transiciones equivalen a 75\% de personas de sexo biológico masculino que transitan al sexo femenino, mientras que el $25 \%$ restante lo conforman hombres cuyo rol de género es el femenino.

De esta forma, para contribuir con el quehacer del logopeda es relevante estudiar una población como ésta, debido a que quienes transitan de un género a otro realizan un enorme esfuerzo para simular una voz que coincida con su identidad de género, lo que podría provocar alteraciones vocales que pueden prevenirse o rehabilitarse mediante terapias complementarias con técnicas y ejercicios vocales adecuados. Por otra parte, según el movimiento de integración y liberación homosexual (MOVILH) de Chile, existe una cifra importante de personas transexuales en este país y a pesar 
de no existir información sobre la prevalencia de esta población, se estima que en relación a las cifras otorgadas por la asociación World Professional Association for Transgender Health (WPATH) y, considerando datos del Censo del año 2012 en Chile (Instituto Nacional de Estadística [INE], 2013) habría una población chilena aproximada de 266 hombres transexuales y 717 mujeres transexuales.

\section{Proceso de modificación de la voz en población transexual}

La voz es parte de las características sexuales de cada individuo, tanto para personas cisgénero como para personas transexuales (Cobeta, Núñez y Fernández, 2013). En individuos transexuales, la voz afecta la vida e identidad de género, debido a que existe dificultades para producir una voz que se asocie al sexo con el que se identifica el sujeto (Cobeta et al., 2013). De acuerdo a Davies y Goldberg (2006), una manera que permite mejorar la salud psicológica y la calidad de vida en personas transexuales es modificar los aspectos de la comunicación que les facilite el proceso de la identidad de género. Para el proceso de masculinización o feminización de la voz, las personas transexuales necesitan de la atención multidisciplinaria de profesionales del área de la salud, entre ellos, el logopeda.

La terapia logopedia proporciona mecanismos que permiten una feminización de la voz de manera funcional y, si se realiza luego de una intervención quirúrgica, ayuda a la recuperación y a una mejor adaptación a la nueva voz resultante (Sauca, 2018). De esta forma, sería igualmente útil en el proceso de masculinización de la voz.

Para Astudillo (2016), los objetivos que se deben tener en cuenta a corto plazo para la modificación de la voz son: conseguir la relajación plena de la musculatura, fortalecer el apoyo abdominal, fortalecer la gestión del soplo, establecer una respiración de tipo costo-diafragmática, instalar una postura y un equilibrio corporal correctos y enseñar sobre la propiocepción, la toma de conciencia del cuerpo y los movimientos respiratorios y fonatorios. En cuanto a los objetivos a largo plazo, éstos consisten en ampliar la extensión vocal, llevar la laringe a efectuar movimientos más amplios y flexibles, favorecer las modulaciones de frecuencias en el habla, reforzar la naturalidad y reducir la artificialidad y, finalmente, transferir lo adquirido a la vida cotidiana (Rodríguez, 2017). Según Astudillo (2016), se consideran necesarias dos sesiones semanales, que varíen en duración según el avance del propio paciente (30, 45 ó 60 minutos) y en las que se trabajen aspectos como la relajación, la respiración, la visualización, la propiocepción, la postura, la gestión del soplo, la prosodia, la articulación, el ritmo, la resonancia, los reflejos naturales, los estados emocionales, etc.

Autores como Rodríguez (2017) y Lesslye (2017) coinciden en que la modificación de la voz de las personas transexuales radica en el aumento o descenso de la F0 y, por tanto, consideran que esta modificación debe ser el objetivo principal del tratamiento fonoaudiológico. La frecuencia fundamental de la mujer se encuentra en un rango entre los 145 y los $275 \mathrm{~Hz}$. y la de los hombres, entre 80 y $165 \mathrm{~Hz}$. Esta diferencia, por lo general, es considerada suficiente para percibir la voz como femenina o como masculina. Para que la voz de un hombre sea reconocida como una voz femenina, debe conseguirse una F0 por encima de 150-160 Hz. y la terapia logopedia por si sola puede lograr un aumento de hasta $40 \mathrm{~Hz}$. Sin embargo, con el paso del 
tiempo, esta nueva voz puede descender o volver a la voz original en situaciones de la vida diaria como al bostezar, toser o reír (Gómez-Raya, 2018). De la misma manera, Alves (2018) refiere que si bien esta terapia genera cambios en la frecuencia fundamental de las personas transexuales, aún hay dificultad en el mantenimiento de la estabilidad de esta frecuencia en el largo plazo.

\section{Terapia de tracto vocal semiocluido}

Las cavidades oral, nasal, faríngea y laríngea componen el tracto vocal donde se encuentran los órganos articulatorios (Cobeta et al., 2013). El tracto vocal actúa como filtro acústico en la producción vocal y cada configuración implica una variación del sonido percibido (Guzmán, 2012).

Story, Laukkanen y Titze (2000) sostienen que para descender las frecuencias de todos los valores formánticos existen dos formas básicas: producir un alargamiento del tracto vocal mediante un descenso laríngeo, una protrusión labial o ambas; y descender los valores formánticos a través de un estrechamiento en los labios, ya que el valor de los formantes desciende a medida que éstos se unen.

La terapia de tracto vocal semiocluido (TVSO) comprende ejercicios cuyo objetivo es lograr un equilibrio fisiológico de los subsistemas involucrados en la producción de la voz: respiración, fonación y resonancia. Según Guzmán, Higueras, Fincheira, Muñoz y Guajardo (2012), la terapia de TVSO beneficia la fonación, logrando el cambio en el patrón vibratorio de los pliegues vocales. Sus principales efectos se relacionan con un aumento de interacción fuente-filtro, una oscilación de pliegues vocales levemente abducidos por la presión retrorefleja del tracto vocal, una disminución en el choque entre los pliegues vocales, una voz más eficiente y económica, una amplificación de sensación de vibración interna y presiones elevadas en el tracto vocal por aumento de presión subglótica. Esto influye en los músculos espiratorios pues se logra mayor activación, especialmente de la musculatura abdominal, lo que permite mantener un apoyo respiratorio más seguro y una producción más balanceada entre el flujo y la presión de aire (Farias, 2007).

Story et al. (2000) investigaron dos técnicas: la fricativa labial y la fonación en un tubo de pequeño diámetro que se colocaba en los labios. Los resultados evidenciaron un aumento de la impedancia de entrada en la frecuencia fundamental, ya que descendía la frecuencia del primer formante. La técnica del fricativo bilabial, por su parte, demostró una disminución en la frecuencia del primer formante y un aumento de la impedancia de frecuencias bajas; sin embargo, la terapia de tubos evidenció mayor eficacia.

Otras investigaciones fueron las realizadas por Gaskill y Erickson (2008), quienes descubrieron los cambios del cociente de contacto (CQ) que se daba por la vibración labial, en donde se mostró una reducción. Sampaio, Oliveira y Behlau (2008) estudiaron los efectos de dos ejercicios: finger kazoo y fonación con tubo. Los resultados fueron positivos y se logró disminuir la F0, pero la valoración perceptivo-auditiva resultó únicamente en la fonación con tubos. Los autores además demostraron que la terapia de tracto vocal semiocluido tenía resultados positivos e inmediatos en la valoración acústica y autovaloración perceptual de la voz.

Laukkanen, Lindholm, Vilkman, Haataja y Alku (1996) comprobaron el efecto de producir el fricativo bilabial / $\beta$ / alternado con la producción de la vocal /a/ y de- 
mostraron que la laringe estaba más baja en la producción de $/ \beta /$, a diferencia de /a/. Además, la emisión de la vocal generó menor actividad muscular.

Por último, Lesslye (2017) en su reciente estudio en Universidad de Washington entrenó a cantantes transexuales. Dentro de los hallazgos, se determinó que los ejercicios de tracto vocal semiocluido eran efectivos para lograr estabilidad de la voz, que está en proceso de cambio. Esto ocurre tanto en la feminización como en la masculinización de la voz por medio de la emisión de tonos cómodos para, luego, moverse a los extremos deseados. Estos efectos se producen de forma inmediata tras realizar una ronda de ejercicios y basta solo una sesión para ver resultados (Farias, 2007).

\section{Materiales y método}

Esta investigación es de tipo cuantitativo, preexperimental, con un diseño de preprueba/posprueba con un solo grupo. A pesar de que en estos diseños el grado de control es mínimo al no existir un grupo de comparación para observar el efecto del tratamiento, al realizarse una medición inicial de la variable depediente se obtiene un punto de referencia del grupo antes del tratamiento (Hernández, Fernández y Baptista, 2010). Específicamente, en este estudio se medirán los parámetros acústicos (variable dependiente) a un solo grupo antes y después de la terapia tracto vocal semiocluido (variable independiente).

El alcance analítico es de tipo descriptivo, debido a que se describen los parámetros acústicos de los participantes transexuales antes y después de la terapia y, a la vez, éstos se comparan para establecer diferencias significativas entre las mediciones pre y postratamiento.

\section{Muestra}

La muestra es de tipo no probabilística, constituida por 8 personas transexuales que transitan del sexo femenino al masculino. Los participantes debían cumplir con criterios de inclusión y exclusión, dentro de los cuales destaca la edad (entre los 13 a 24 años) y la ausencia de cualquier tratamiento realizado con el propósito de modificar la voz.

\section{Instrumentos}

La recopilación de información se realiza mediante la utilización de anamnesis, la aplicación del Programa de Análisis Acústico PRATT (Boersma y Weenink, 2018) y el instrumento Voice Handicap Index (VHI, Núñez-Batalla et al., 2007). Esto último solo se aplicó con las finalidad de decribir la percepción de los participantes en relación a su voz antes de la terapia. Sin embargo, es importante señalar que la finalidad de este estudio no fue medir la autopercepción de la población transexual, sino focalizarse en el efecto inmediato de la terapia de TVSO sobre los parámetricos acústicos.

El programa PRAAT es un software que se utiliza para analizar, sintetizar y manipular señales de habla que procesa muestras de habla y entrega información acústica 
como espectrogramas de banda ancha y estrecha, espectros, formantes, frecuencia fundamental (F0), intensidad, duración, relación armónico-ruido, entre otros. Este instrumento cuenta con la validez y confiabilidad en un 100\% (Cecconello, 2008).

Por otro lado, el VHI es un cuestionario desarrollado con el fin de cuantificar el impacto percibido por un sujeto afectado por un trastorno vocal en los ámbitos de la propia función vocal, en la capacidad física relacionada con ella y en las emociones que provoca su afectación (Jacobson et al., 1997). Cuenta con treinta preguntas divididas en estas tres partes relacionadas con información funcional, físico y emocional. En este instrumento se categorizan los puntajes totales en leve (puntaje de 0 a 30 puntos), moderado (puntaje de 31 a 60 puntos), severo (puntaje de 61 a 90) y grave (puntaje de 91 a 120 puntos) (Núñez-Batalla et al., 2007).

\section{Procedimiento}

En primer lugar, se les solicita a los participantes firmar un Consentimiento Informado, el cual informa sobre el procedimiento y los objetivos del estudio, además de asegurar la confiabilidad de los antecedentes personales y de los resultados del estudio. Cabe señalar que este documento, junto con el proyecto, fue validado por un Comité de Ética 5 .

Luego se procede a aplicar la anamnesis y realizar las evaluaciones mediante el VHI y el PRAAT. La grabación del PRAAT se realiza en un computador con el software previamente instalado, utilizando un micrófono que abarque un rango de frecuencia entre 20 a $20000 \mathrm{~Hz}$. El lugar de aplicación es un espacio físico donde el ruido ambiente no supere los 25 decibeles. Al momento de grabar, los participantes del estudio deben estar sentados con la espalda afirmada en el respaldo de la silla, los pies apoyados en el suelo sin estar cruzados y los brazos a los lados. Quienes componen esta muestra debieron enunciar una /a/ sostenida en tono medio, hablando a una distancia de 15 a 20 centímetros aproximadamente del micrófono. A partir de dicha grabación, realizada antes y después de la terapia, se obtienen los datos para el análisis de los parámetros acústicos. Si bien la literatura valida dos técnicas para la medición de parámetros acústicos que son la voz hablada y la vocal sostenida, esta investigación optó por esta última ya que permite tener mayor control sobre la emisión de la vocal. Estudios como los realizado por Núnez-Batalla, Díaz-Molina, García-López, Moreno-Méndez, Costales-Marcos, Moreno-Galindo y Martínez-Camblor (2012) y Hernández, Gómez, Ruiz y Arteaga (2017) avalan la utilización de este método.

El ejercicio de Tracto Vocal Semiocluido consistió en la fonación en tubos, que comprendía fonar en un tubo produciendo el fonema $/ \beta /$, primero en forma sostenida, y, luego, en glissandos descendentes. Finalmente, se repetía la fonación en tubo pero con resistencia: fonar en tubo en un vaso con agua. Los ejercicios con tubos de resonancia son beneficiosos, pues favorece la economía vocal y se pueden cumplir altas exigencias sin correr el riesgo de causar daño mecánico en los pliegues vocales (Cecconello, 2009). La terapia de TVSO, en general, beneficia el cambio de patrón vibratorio en los pliegues vocales.

La duración de la terapia es de aproximadamente 20 minutos por sujeto. 


\section{Resultados}

Los siguientes son los resultados que permiten describir el efecto inmediato de los parámetros acústicos a partir de la terapia de tracto vocal semiocluido en personas transexuales de 13 a 24 años.

El primer análisis realizado corresponde a una estadística descriptiva con la finalidad de, por un lado, conocer la distribución de la muestra en cuanto a edad y, por otro, caracterizar la percepción de los participates en cuando a su voz y los parámetros acústicos antes y después de la terapia. Posteriormente, se realiza un análisis inferencial con el propósito de determinar las diferencias significativas entre los parámetros acústicos pre y post-terapia.

La tabla 1 muestra los datos relacionados con la edad de los participantes:

Tabla 1: Descripción de la muestra según la edad.

\begin{tabular}{ccccc}
\hline $\mathrm{N}^{\mathbf{0}}$ & Media Edad & DS & Máxima & Mínima \\
\hline 8 & 19 & 3,7 & 24 & 13 \\
\hline
\end{tabular}

Como se observa en la tabla 1, los participantes se encuentran entre los 13 y 24 años, presentando una media de 19 años, edad adecuada para la evaluación ya que están fuera del período de la muda vocal (período de cambio de voz a causa de la pubertad), salvo el participante de 13 años. Este individuo, en particular, presentó valores en los parámestros acústicos muy similares en relación a los demás participantes, por lo cual no fue excluído debido a que no debiese ser una condición que influya en los resultados.

En cuanto a los resultados asociados a la percepción de los parámetros vocales, éstos se muestran en la siguiente tabla:

Tabla 2: Puntaje del VHI antes de la terapia tracto vocal semiocluido.

\begin{tabular}{cccccc}
\hline & N & Mínimo & Máximo & Media & Desv. típ. \\
\hline VHI funcional & 8 & 7 & 30 & 16,88 & 7,74 \\
VHI física & 8 & 10 & 27 & 18,50 & 4,99 \\
VHI emocional & 8 & 11 & 35 & 22,88 & 8,64 \\
VHI Total & 8 & 28 & 81 & 58,25 & 18,03 \\
\hline
\end{tabular}

De acuerdo a la tabla 2, los participantes perciben mayor afectación en el aspecto emocional asociado a su voz, luego a su capacidad física y, finalmente, en lo que respecta a la función vocal. De acuerdo a la media obtenida en cada subprueba del VHI, las personas transexuales que conforman este estudio se encontrarían con una discapacidad leve en los aspectos funcional y físico y moderada en cuanto a su percepción emocional; mientras que el puntaje total obtenido en el VHI los categoriza en una discapacidad moderada. Lo anterior, puede observarse en la siguiente tabla 3 que indica la frecuencia encontrada en la discapacidad de cada una de las subevaluaciones del instrumento: 
Tabla 3: Resultados categóricos del VHI antes de la terapia.

\begin{tabular}{ccccc}
\hline & Funcional & Físico & Emocional & VHI Total \\
\hline Leve & 5 & 5 & 2 & 1 \\
Moderado & 3 & 3 & 5 & 3 \\
Severo & 0 & 0 & 1 & 4 \\
Total & 8 & 8 & 8 & 8 \\
\hline
\end{tabular}

Como se observa en la tabla anterior, el $62,5 \%$ de la muestra evidencia una discapacidad leve y el 37,5\% se ubica dentro de la categoría de moderado tanto en los apectos funcional y físico de la voz. Cabe señalar que no hay participantes que se encuentren dentro de la categoría severo ni grave en estas mediciones. En cuanto al aspecto emocional asociado con su discapacidad vocal, el $25 \%$ de la muestra se encuentra en la categoría leve, el $62,5 \%$ en la categoría moderado y $12,5 \%$ refiere tener una discapacidad severa. La interpretación del puntaje total de VHI indica que el $12,5 \%$ de la muestra evidencia una discapacidad leve, mientras que $37,5 \%$ se ubican dentro de la categoría asociada a la discapacidad moderada y el 50\% refiere tener una discapacidad severa.

En cuanto a los parámetros acústicos medidos antes y depués de la terapia vocal, la tabla 4 muestra los valores obtenidos en cada uno de ellos:

Tabla 4: Descripción de los parámetros acústicos antes y después de la terapia.

\begin{tabular}{ccccc}
\hline $\begin{array}{c}\text { Parámetro } \\
\text { Acústico }\end{array}$ & $\begin{array}{c}\text { Media antes } \\
\text { de la terapia }\end{array}$ & DS & $\begin{array}{c}\text { Media después } \\
\text { de la terapia }\end{array}$ & DS \\
\hline jitter (local) & 0,58 & 0,29 & 0,32 & 0,17 \\
jitter (rap) & 0,34 & 0,18 & 0,17 & 0,096 \\
jitter (ppq5) & 0,33 & 0,15 & 0,21 & 0,11 \\
shimmer (local) & 7,15 & 6,49 & 5,31 & 2,28 \\
shimmer (apq3) & 3,63 & 3,34 & 2,15 & 0,95 \\
shimmer (apq11) & 6,02 & 3,97 & 5,75 & 2,26 \\
F0 & 186,49 & 37,56 & 177,62 & 33,71 \\
HNR & 15,20 & 7,27 & 19,79 & 4,56 \\
\hline
\end{tabular}

La tabla anterior evidencia que cada uno de los parámetros medidos disminuye sus valores después de la terapia, con excepción de HNR. El parámero acústico Jitter evidencia las siguientes diferencias de puntaje entre las mediciones inicial y final: Jitter Local tiene una diferencia de 0,26 puntos; Jitter Rap, de 0,17; y Jitter ppq5, de 0,12 . El Shimmer muestra las siguientes variaciones: en Shimmer local, la diferencia es de 1,84; Shimmer apq3 varían en 1,48; y el Shimmer apq11 muestra diferencias de 0,27. La Frecuencia Fundamental (F0) presenta una variación de 8,87 entre las mediciones antes y después de la terapia, lo que indicaría que la voz de las personas transexuales se hizo más grave. La Relación o Señal Armónico Ruido muestra una diferencia de puntaje de 4,59 por el efecto de la terapia tracto vocal semiocluido; corresponde al único parámetro que sufrió un aumento en sus valores, lo que se considera positivo ya que se acercó a los parámetros de normalidad que equivale a $20 \mathrm{db}$. 
Ahora bien, con la finalidad de establecer diferencias significativas entre los parámetros acústicos medidos antes y después de la terapia en la muestra, los datos fueron sometidos a la prueba de distribución Shapiro-Wilk, ya que la muestra es pequeña y no se cumplen los supuestos de una distribución normal. De acuerdo a los resultados que arroja este estadígrafo, la comparación de medias fue realizada con la prueba T-student para muestras relacionadas debido a que los parámetros acústicos mostraron una distribución normal, exceptuando la F0 que fue analizada con la prueba para muestras relacionadas no paramétrica Wilcoxon.

A continuación se observan los resultados obtenidos a partir de la comparación de medias en los parámetros acústicos distribuidos de manera normal:

Tabla 5: Comparación de los parámetros acústicos antes y después de la terapia según la prueba $\mathrm{T}$ Student para muetras relacionadas.

\begin{tabular}{lcc}
\hline & $\mathrm{t}$ & Sig. (bilateral) \\
\hline jitter (local) antes - jitter (local) después & 2,549 & $0,038^{*}$ \\
jitter (rap) antes - jitter (rap) después & 2,638 & $0,034^{*}$ \\
jitter (ppq5) antes - jitter (ppq5) después & 2,281 & 0,057 \\
shimmer (local) antes - Shimmer (local) después &, 733 & 0,487 \\
shimmer (apq3) antes - Shimmer (apq3) después & 1,196 & 0,271 \\
shimmer (apq11) antes - Shimmer (apq11) después &, 151 & 0,884 \\
HNR antes - HNR después &, 956 & 0,371 \\
\hline
\end{tabular}

A partir de la tabla anterior, se observa que existen diferencias estadísticamente significativas entre las evaluaciones correpondientes a antes y después de la terapia en el Jitter Local y el Jitter Rap con un nivel de significación de 0,05 . Esto es, la terapia de tracto vocal semiocluido tuvo efectos inmediatos en los parámetros acústicos de Jitter Local y Rap. El Jitter ppq5 muestra diferecias pero éstas no llegan a ser significativas, al igual que los otros parámetros acústicos evaluados.

En cuanto a la Frecuencia Fundamental, los resultados de la prueba estadística Wilcoxon se muestran en la tabla 6:

Tabla 6: Comparación de la frecuencia fundamental antes y después de la terapia según la prueba Wilcoxon.

\begin{tabular}{|c|c|}
\hline & F0 después $-\mathrm{F} 0$ antes \\
\hline$Z$ & $-1,540 \mathrm{~b}$ \\
\hline Sig. asintót. (bilateral) & 0,123 \\
\hline
\end{tabular}

Como se observa en la tabla 6 , los resultados arrojan un valor no significativo por lo que se establece que no existen efectos inmediatos de los ejercicios de TVSO que establezcan diferencias estadísticamente significativas en la frecuencia fundamental. Sin embargo, es importante considerar que el hecho de que el valor de este parámetro haya disminuido, significa que la voz de las personas transexuales se hizo más grave. 


\section{Discusión}

Este estudio permitió determinar el efecto de la terapia de tracto vocal semiocluido sobre los parámetros acústicos de 8 personas que transitan del sexo femenino a masculino de 13 a 24 años. Los resultados indican que, a pesar de que todos los parámetros acústicos evaluados evidenciaron mejoría luego de la terapia, ésta sólo tuvo efecto inmediato sobre el Jitter Local y el Jitter Rap, puesto que estos parámetros mostraron una diferencia estadísticamente significativa en sus valores antes y después de la terapia. Lo anterior coincide sólo parcialmente con el estudio de Barrera y Pezo (2017), en el que los parámetros de Shimmer y extensión tonal se vieron aumentados y disminuidos (dependiendo de la transición de género) en relación a los valores normales.

Por otra parte, en relación a la Frecuencia Fundamental, si bien no hubo diferencia significativa entre los valores pre y post terapia, se evidencia una disminución en el valor de ésta, lo que indica que la voz de los individuos se hizo más grave posterior a la terapia. Esta variación responde a características fisiológicas de la voz y no es una conducta forzada, lo cual indica que la TVSO tuvo un efecto fisiológico. Sin embargo, cabe preguntarse si el efecto es sostenido en el tiempo o se requiere de un mayor número de sesiones terapéuticas. El hecho de que la F0 no muestre variación significativas entre sus valores antes y después de la terapia puede deberse a lo planteado por Rodríguez (2017), quién menciona que si bien hay personas transexuales que logran masculinizar su voz con terapia, otros no lo consiguen.

En relación al parámetro HNR, éste se acercó mayormente al valor de normalidad posterior a la terapia, resultados que se condicen con lo planteado por Vásquez, Retalmal y Zapata (2016), quienes obtuvieron resultados similares en cuanto a la mejoría de este parámetro post aplicación de las técnicas de TVSO, consiguiendo aumentar la intensidad de los armónicos por sobre el ruido en la emisión de una /a/ sostenida.

Autores como Guzmán et al. (2012), Sampaio et al. (2008) y Titze (2006) avalan el efecto inmediato de la terapia de TVSO producto de las modificaciones que sufren los parámetros acústicos luego de su aplicación. No obstante, debido a que en esta población la terapia mostró diferencias estadísticamente significativas sólo en el Jitter en sus mediciones pre y post-terapia, se sugiere la incorporación de un mayor número de sesiones para que los efectos sean mayores y éstos logren matenerse en el tiempo.

En esta muestra en particular, no se consideró la afectación de parámetros acústicos debido a que éstos puediesen estar más afectados o perturbados porque las personas que conforman esta muestra están incurriendo en conductas de abuso y mal uso vocal al intertar forzar su voz para asemejarse a la ideal.

A pesar de que el VHI es un instrumento que permite medir la autopercepción para voces patológicas, la mitad de esta muestra refiere tener un discapacidad vocal severa. Por esta razón, se propone que próximas investigaciones utilicen el cuestionario Transsexual Voice Questionnaire for Male-to-Female Transsexuals (TVQ ${ }^{\mathrm{MtF}}$ ) de Dacakis, Davies, Oates, Douglas y Johnston (2013), en su versión en española (Mora, Carrillo, Giribet, Becerra, Lucio y Cobeta, 2018) para este tipo de muestra, con la salvedad de que este instrumento se aplica a quienes transitan del género masculino a femenino. Es por ello que es importante destacar la necesidad de una adaptación de este cuestionario para las personas que transitan del género femenino al masculino debido a que es una población que va en aumento y son vulnerables a 
patologías vocales (al igual que aquellos que transitan del género masculino al femenino) por el esfuerzo que realizan para aproximarse a la voz del género con el cual se sienten identificados.

\section{Conclusión}

La terapia de traco vocal semiocluido fue eficaz en el proceso de masculinización de la voz en personas transexuales que participaron en este estudio, a pesar de que los resultados obtenidos sólo arrojan diferencias estadísticamente significativas en el parámetro Jitter. La terapia de TVSO tuvo efectos positivos debido a que parámetros de perturbación como el Jitter y el Shimmer mostraron modificaciones al disminuir en una sóla sesión de terapia, lo cual indica que existe menos perturbación en la voz; la F0 también disminuyó, lo que indica que la voz de estas personas transexuales que transitan del género femenino al masculino se hizo más grave; y el HNR aumentó y se aproximó a los valores normativos. No obstante, la incorporación de un mayor número de sesiones podría tener un mayor efecto sobre los parámetros evaluados, permitiendo tener significancia entre sus valores antes y después de la terapia.

Cabe destacar que los métodos para el proceso de masculinización de la voz más recurrentes son la hormonoterapia; sin embargo, es un método invasivo y costoso, por lo que una terapia logopedia como la realizada en el presente estudio puede ser un alternativa complementaria para la población transexual con el fin de mejorar su calidad de vida. Particularmente en esta muestra, la terapia de TVSO se mostró como una alternativa viable en el proceso de masculinización de la voz de personas transexuales, considerando que estos individuos están sujetos a continuas conductas de abuso y mal uso vocal por la necesidad de modificar voluntariamente sus características vocales con el fin de acercarlas a los parámetros de su identidad de género. Esto conlleva a esfuerzos y hábitos nocivos que repercuten sobre la salud vocal.

En base a lo anterior, los ejercicios de TVSO actuaron positivamente modificando los parámetros acústicos de la voz por medio de cambios fisiológicos del sistema fonatorio, lo cual abre la alternativa al uso de esta terapia con esta población, con el fin de lograr cambios reales y sustanciales de las características vocales asociadas al sexo biológico de cada individuo.

A partir de lo observado, surge la inquietud sobre la durabilidad del cambio en los parámetros acústicos, ya que sólo se observó el efecto inmediato posterior a una sesión de terapia. Cabe preguntarse, entonces, si con una terapia vocal más prolongada estos cambios se hacen permanentes, cuántas sesiones son las indicadas para establecer dichos cambios y en qué edades es oportuno intervenir. Por último, ante estas interrogantes sería necesario establecer valores de normalidad de los parámetros acústicos en personas transexuales, considerando evidentemente su proceso de transición: feminización o masculinización de la voz.

\section{Referencias bibliográficas}

Alves, J. S. D. (2018). Efeitos da terapia fonoaudiológica na voz de mulheres transexuais. Bachelor's thesis, Universidade Federal do Rio Grande do Norte. 
Astudillo, M. (2016). La feminización de la voz de la mujer transexual. España: Círculo Rojo. Boersma, P., y Weenink, D. (2018). Praat: doing phonetics by computer [Computer program]. Version 6.0.40, revisado el 11 May de 2018 de http://www.praat.org/

Casado, J. C. (2002). La evaluación de la voz: Fundamentos médicos y logopédicos. Málaga: Aljibe.

Cecconello L. (2009). Ejercicios de tracto vocal semiocluido. XII Jornadas Foniátricas, Universidad Nacional de San Luis, Facultad de Ciencias Humanas, San Luis: Argentina, 2009, CD ROM, ISBN 978-987-1595-02-0.

Cecconello, L. (2008). Manual básico para grabación y análisis de la voz. Valencia: España. Cobeta, I. (2013). Patología de la voz. Edición Marge Médica Books. Barcelona: España.

Cobeta, I., Nuñez, F., y Fernández, S. (2013). Patología de la Voz. Barcelona: Marge Médica Books.

Dacakis, G., Davies, S., Oates, J. M., Douglas, J. M., y Johnston, J. R. (2013). Development and preliminary evaluation of the transsexual voice questionnaire for male-to-female transsexuals. Journal of Voice, 27(3), 312-320.

Davies, S., y Goldberg, J. (2006). Transgender Speech Feminization/Masculinization: Suggested Guidelines for BC Clinicians. Vancouver Coastal Health, Transcend Transgender Support \& Education Society, \& the Canadian Rainbow Health Coalition.

Elisei, N. G. (2012). Análisis acústico de la voz normal y patológica utilizando dos sistemas diferentes: ANAGRAF y PRAAT. Interdisciplinaria, 29(2), 271-286.

Farias P. (2007). Ejercicios que restauran la función vocal-1a ed. Buenos Aires: Libreria Akadia.

Gaskill, C. S., y Erickson, M. L. (2008). The effect of a voiced lip trill on estimated glottal closed quotient. Journal of Voice, 22(6), 634-643.

Gómez-Raya, A. (2018) Intervención logopédica en la feminización de la voz en transexuales: revisión bibliográfica. Revista de Investigación en Logopedia. 8(1) 2018, 21-42.

Guzmán, M. (2012). Terapia con tracto vocal semi-ocluido: un estudio de caso. Revista Chilena de Fonoaudiología 11, 87-97.

Guzmán, M., Higueras, D., Fincheira, C., Muñoz, D., y Guajardo, C. (2012). Efectos acústicos inmediatos de una secuencia de ejercicios vocales con tubos de resonancia. Revista CEFAC 14(3), 471- 480.

Hernández, R., Fernández, C., y Baptista, P. (2010). Metodología de la investigación. Ciudad de México: Mc Graw Hill.

Hernández, J. D., Gómez, N. M. L., Ruiz, A. J., y Arteaga, L. I. (2017). Análisis acústico de la voz: medidas temporales, espectrales y cepstrales en la voz normal con el Praat en una muestra de hablantes de español. Revista de Investigación en Logopedia, 7(2), 108-127.

Instituto Nacional de Estadísticas [INE] (2013). Informe final comisión externa revisora del CENSO 2012. Recuperado en http://historico.ine.cl/canales/chile_estadistico/censos_poblacion_vivienda/comision_investigadora/nacional/anexos_comision_externa_revisora-comision-nacional.pdf

Jackson-Menaldi, M. C. A. (2002). La voz patológica. Ed. Médica Panamericana.

Jackson-Menaldi, M. C. A. (1992). La voz normal. Ed. Médica Panamericana.

Jacobson, B. H., Johnson, A., Grywalski, C., Silbergleit, A., Jacobson, G., Benninger, M. S., y Newman, C. W. (1997). The voice handicap index (VHI): development and validation. American Journal of Speech-Language Pathology, 6(3), 66-70.

Laukkanen, A. M., Lindholm, P., Vilkman, E., Haataja, K., y Alku, P. (1996). A physiological and acoustic study on voiced bilabial fricative/ $\beta$ :/as a vocal exercise. Journal of Voice, 10(1), 67-77. 
Lessley, E. D. H. (2017). Teaching Transgender Singers. Tesis Doctoral.

Lopes, J., Freitas, S., Sousa, R., Matos, J., Abreu, F. y Ferreira, A. (2008). A medida HNR: sua relevância na análise acústica da voz e sua estimação precisa. Procedings of I Jornadas sobre Tecnologia e Saúde, Guarda, Portugal.

Mora, E., Carrillo, A., Giribet, A., Becerra, A., Lucio, M. J., y Cobeta, I. (2018). Translation, cultural adaptation, and preliminary evaluation of the Spanish version of the transgender voice questionnaire for male-to-female transsexuals (TVQ MtF). Journal of Voice, 32(4), 514-e1

Núñez-Batalla, F., Corte-Santos, P., Señaris-González, B., Llorente-Pendás, J. L., Górriz-Gil, C., y Suárez-Nieto, C. (2007). Adaptación y validación del índice de incapacidad vocal (VHI-30) y su versión abreviada (VHI-10) al español. Acta Otorrinolaringol Española, 58(9), 386-392. doi:10.1016/S0001-6519(07)74954-3

Núnez-Batalla, F., Díaz-Molina, J. P., García-López, I., Moreno-Méndez, A., Costales-Marcos, M., Moreno-Galindo, C., y Martínez-Camblor, P. (2012). El espectrograma de banda estrecha como ayuda para el aprendizaje del método GRABS de análisis perceptual de la disfonía. Acta Otorrinolaringológica Española, 63(3), 173-179.

Pérez, A. (2017). Efectividad de un programa de intervención en casos de feminización y masculinización de la voz en personas transgénero. Tesis de pregrado. Universidad de Granada.

Sampaio, M., Oliveira, G., y Behlau, M. (2008). Investigação de efeitos imediatos de dois exercícios de trato vocal semi-ocluído. Pro-Fono Revista de Atualização Científica. 20(4), 261-6. DOI: 10.1590/S0104-56872008000400010

Sauca, A. (2018). Clínica de la veu. Recuperado de: www.asauca.net/voz-transgenero.php

Story, B. H., Laukkanen, A. M., y Titze, I. R. (2000). Acoustic impedance of an artificially lengthened and constricted vocal tract. Journal of Voice, 14(4), 455-469.

Titze I. (2006). Voice training and therapy with a semi- occluded vocal tract: rationale and scientific underpinnings. Journal Speech, Language and Hearing Research, 49(2), 44859.

Vásquez, K. , Retamal, M. P., y Zapata, Y. (2016). Efectos de la terapia de Tracto Vocal Semi-Ocluido sobre los parámetros acústicos de la voz en docentes. Apuntes Universitarios, 6(2), 9-39.

World Professional Association for Transgender Health (WPATH), revisado el 12 de noviembre de 2018 de https://www.wpath.org. 